\title{
16330 \\ Evaluation of four different titanium surface treatment on the bone healing in defects filled only with blood clot: an experimental animal study.
}

\section{BASIC RESEARCH}

\begin{abstract}
Gehrke, Sergio A. 1; Pérez, Leticia 2; Mazon, Patricia 3; De Aza, Piedad N. ${ }^{4}$

${ }^{1}$ Biotecnos - Technology and Science, Montevideo, Uruguay; ${ }^{2}$ Laboratorio de Interacciones Moleculares, UDELAR, Montevideo, Uruguay; ${ }^{3}$ Departamento de Materiales, Óptica y Tecnologia Electrónica, Universidad Miguel Hernández, Elche (Alicante), Spain; 4 Instituto de Bioingenieria. Universidad Miguel Hernández, Elche, (Alicante), Spain.
\end{abstract}

Abstract

Background: Among the modifications in the sense of improving tissue response, surface treatment of implants has received more attention and is one of the most researched topics. Since the first portion of an implant to interact with the patient's tissues after implantation is the surface of the implant where direct contact occurs with the blood and consequently with its cellular components and growth factors. Aim: The aim of the present histologic animal study was to analyze whether roughness of the titanium surface can influence and/or stimulate the bone growth in defects filled with the blood using a rabbit tibia model. Material and Methods: Forty sets (implant and abutment), dental implant $(3.5 \mathrm{~mm}$ in diameter and $7 \mathrm{~mm}$ in length) plus healing abutment $(2.5 \mathrm{~mm}$ in diameter) were inserted in the tibiae of 10 rabbits. Moreover, twenty titanium discs were prepared. The abutment and discs were treated by 4 different methods and divided in 4 groups: (group A) machined abutments (smooth); (group B) double acid etching treatment; (group C) treatment with blasting with particles of aluminum oxide blasted plus acid conditioning (group D) treatment with through blasting with particles of titanium oxide plus acid conditioning. The discs were used to characterize the surfaces by a profilometer and scanning electronic microscopy. Results; After 8 weeks, the new bone formation around the sets of the samples were analyzed qualitative and quantitatively in relation to bone height from the base of the implant and presence of osteocytes. In the group $C(1.50 \pm 0.20 \mathrm{~mm})$ and group $D$ $(1.62 \pm 0.18 \mathrm{~mm})$ showed bone growth on the abutment with higher values in compare to group $\mathrm{A}(0.94 \pm 0.30$ $\mathrm{mm})$ and $B(1.19 \pm 0.23 \mathrm{~mm})$, with significant difference between the groups $(P<0.05)$. In addition, osteocyte presence was higher in groups with surface treatment related to machined $(P<0.05)$. Conclusions: Within the limitations of the present study, it was possible to observe that there is a direct relationship between the roughness present on the titanium surface and the stimulus for bone formation, since the presence of larger amounts of osteocytes on SLA surfaces evidenced this fact. Furthermore, the increased formation of bone tissue in height demonstrates that there is an important difference between the (physical / chemical) methods used for surface treatment

\section{Background and Aim}

Among the modifications in the sense of improving tissue response, surface treatment of implants has received more attention and is one of the most researched topics. Since the first portion of an implant to interact with the patient's tissues after implantation is the surface of the implant where direct contact occurs with the blood and consequently with its cellular components and growth factors, its morphological structure (roughness pattern) and / or physical-chemical characteristics are widely analyzed. The first step for the bone healing on the titanium implant (osseointegration) is forming a blood clot at the surface. The initial contact of blood with biomaterials and subsequent recruitment of inflammatory and marrow-derived stromal cells is among the first phases of bone regeneration. The cellular activities (adhesion and growth) on a surface is influenced directly by its morphological characteristics and its chemical composition. Then, the purpose of this histological animal study was to analyze the effect of titanium surface with different roughness patterns on bone tissue formation in small defects (as a healing chamber) filled only with blood clot in the tibia of rabbits.

\section{Methods and Materials}

Forty sets (implant and abutment), dental implant (3.5 $\mathrm{mm}$ in diamete and $7 \mathrm{~mm}$ in length) plus healing abutment $(2.5 \mathrm{~mm}$ in diameter) were inserted in the tibiae of 10 rabbits. Moreover, twenty titanium discs were prepared. The abutment and discs were treated by 4 different methods and divided in 4 groups: (group A) machined abutments (smooth); (grour B) double acid etching treatment; (group C) treatment with blasting with particles of aluminum oxide blasted plus acid conditioning (group D) treatment with through blasting with particles of titanium oxide plus acic conditioning. The discs were used to characterize the surfaces by a profilometer and scanning electronic microscopy.

\section{Results}

\section{Implants installation and parameters analyzed:}
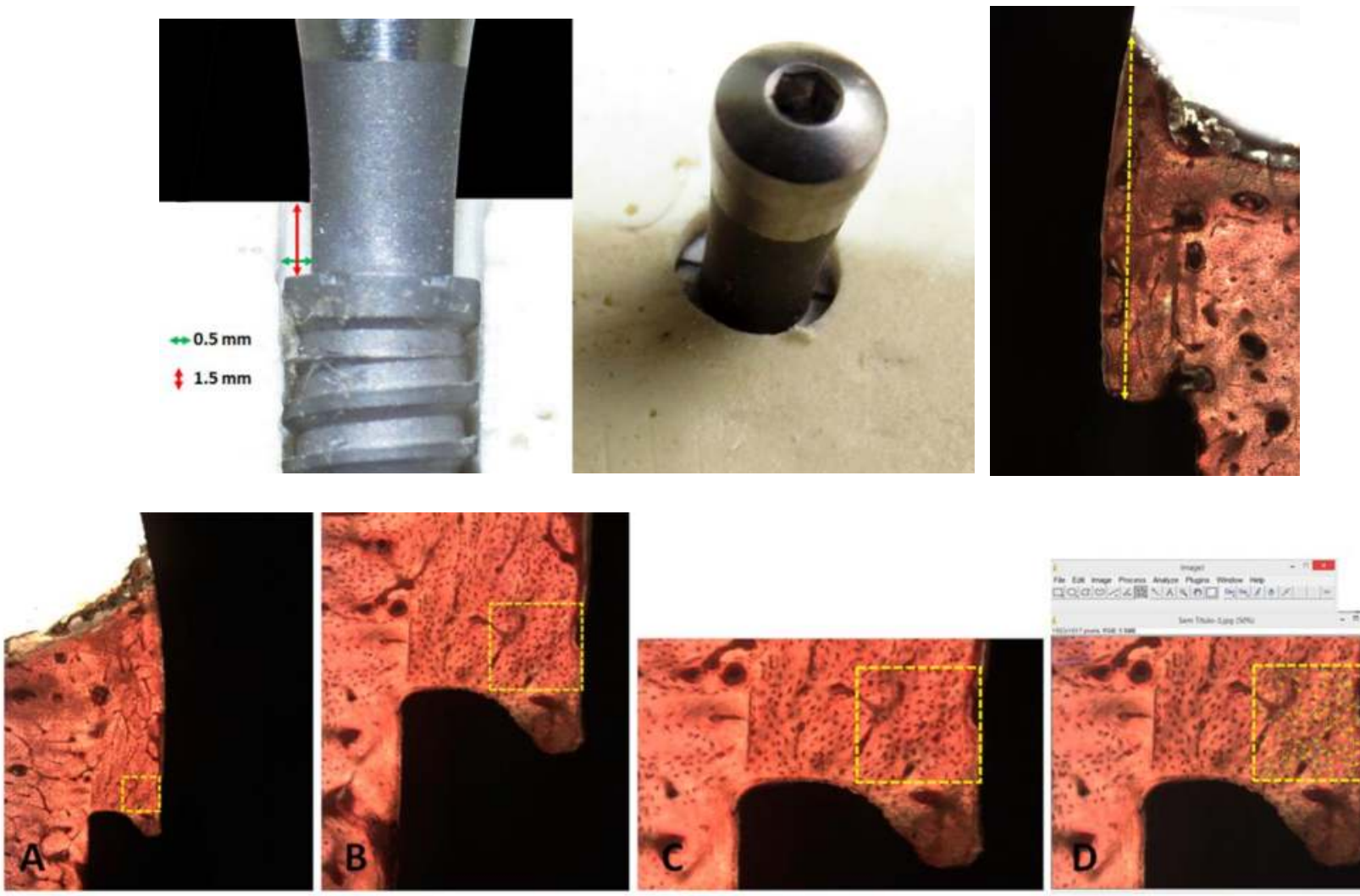

Results

Histomorphological analysis and measurements:

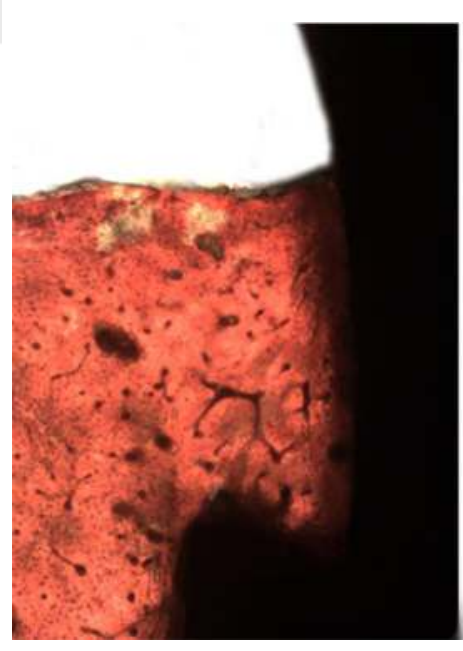

Group A

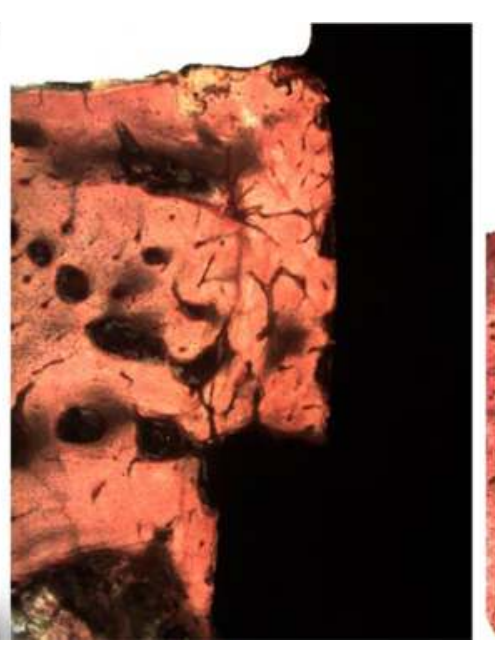

Group B

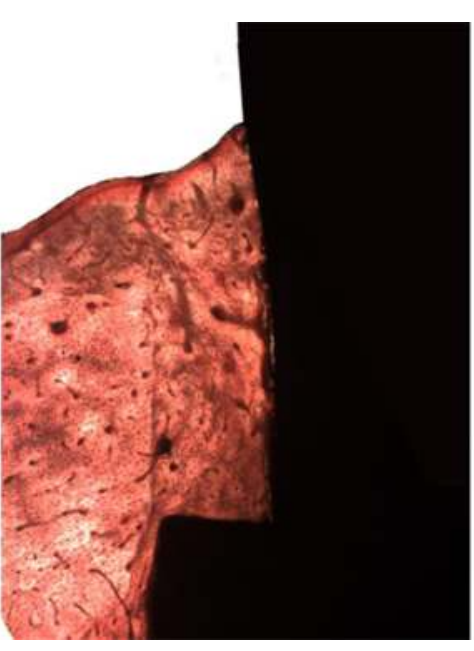

Group C

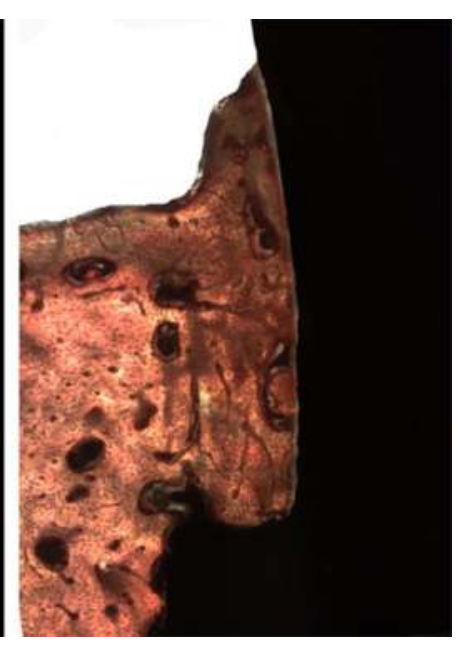

Group D
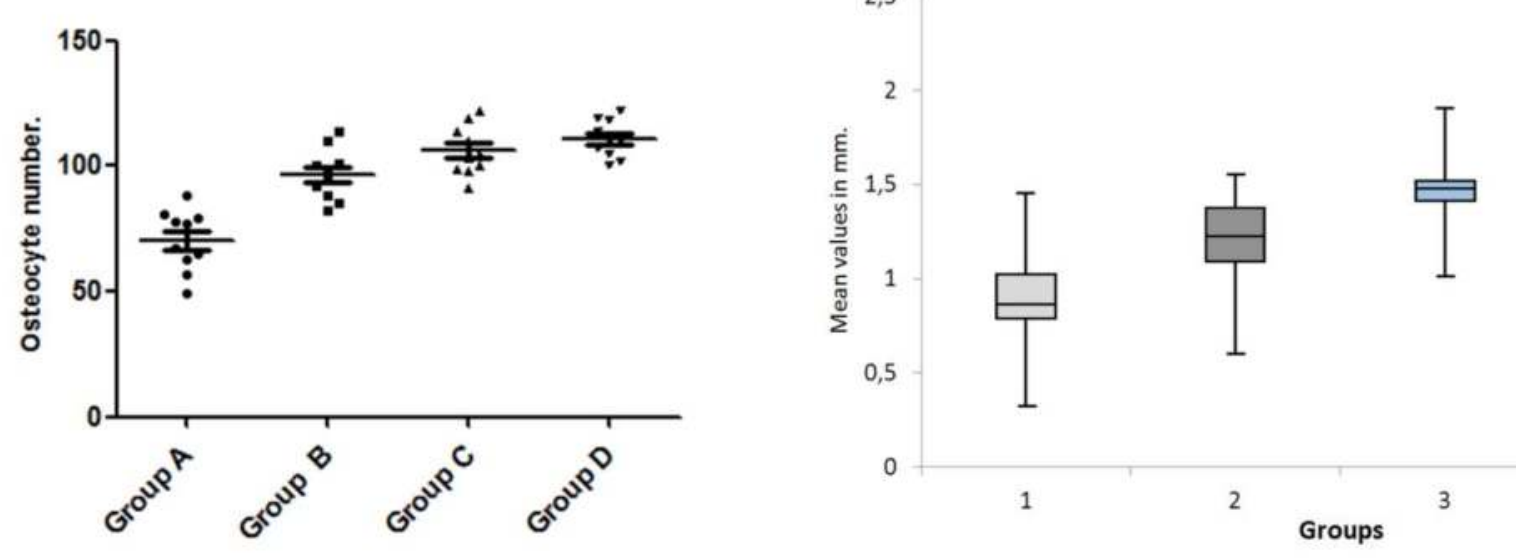

\section{Conclusion}

Within the limitations of the present study, it was possible to observe that there is a direct relationship between the roughness present on the titanium surface and the stimulus for bone formation, since the presence of larger amounts of osteocytes on SLA surfaces evidenced this fact. Furthermore, the increased formation of bone tissue in height demonstrates that there is an important difference between the (physical / chemical) methods used for surface treatment. 